\title{
Calidad y productividad: Un enfoque alternativo
}

\section{García de Berrios, Omaira*}

\section{Resumen}

El propósito de este trabajo es explorar el carácter de las propuestas de Calidad y Productividad. Se parte del supuesto que dicha propuesta es un requerimiento de la nueva organización de la producción, motorizada por la necesidad de competencia, fundamentada en una producción individualizada, que sustituye la producción en masa. El análisis revela que la propuesta de Calidad y Productividad favorece la legitimación de la ideología capitalista en su trasfondo, donde el contenido básico es la racionalidad económica; se ubica dentro del sistema de valores un carácter aplicativo, utilitarista y hedonista; as como se infiere que esta propuesta tiene como fin el posisionamiento en los mercados internacionales. Se concluye que calidad y productividad además de sustentarse en la búsqueda de incremento de la ganancia y expansión del capital es una manifestación ideológica que representa la situación del sistema capitalista como natural y perpetua.

Palabras clave: Calidad y productividad, administración, ideología, sistema capitalista, globalización.

Recibido: $98-10-20$. Aceptado: $99-07-14$

* Profesora de la Universidad de Los Andes (ULA). Participante del Programa de Doctorado en Ciencias Humanas de La Universidad del Zulia (LUZ). 


\section{Quality and Productivity: An Alternative Focus}

\section{Abstract}

The purpose of this research is to explore the character of quality and productivity proposals. These proposals are considered as requirements in modern production organization, motivated by the need to compete, and based on individualized production, which is a substitute for mass production. The analysis reveals that quality and productivity proposals favor the legitimacy of the underpinnings of capitalistic ideology, where the basic content is economic rationality; this is found in a value system of an applied, utilitarian and hedonistic character. It also infers that the goal of this proposal is positioning in international markets. The conclusion is that quality and productivity, apart from justifying themselves in the search for increased earnings and capital expansion, are an ideological manifestation which supposes that the capitalistic system is natural and perpetual.

Key words: Quality and productivity, administration, ideology, capitalist system, globalization.

\section{Introducción}

La relación Calidad y Productividad ha ocupado la atención de la disciplina administrativa de modo intenso desde la década de los ochenta, a raíz de la divulgación por parte de Deming, de algunas propuestas para incrementar la calidad en función de la productividad, a partir de su observación de la introducción de dicha relación en la economía japonesa después de la segunda guerra mundial.

La calidad aparece como la varita mágica para salir de la crisis; una increlble producción intelectual que intenta mostrarnos la mejor forma de encontrar la relación calidad y productividad en cualquier esfera de la vida social, ha invadido el mercado bibliográfico. La oferta de capacitación en materia de calidad ha sido cuantiosa, como respuesta a una deman- da motivada por la necesidad de no quedarse atrás. Vinculado a esto, una intensa actividad de capacitación sobre las propuestas de calidad han tenido lugar desde los años ochenta en casi todos los paises en lo cual se han gastado cuantiosos recursos.

Numerosos intelectuales se ha dedicado a la consultoría de calidad, la mayoría de las empresas y gran número de instituciones del Estado declaran formalmente la realización de esfuerzos en la implantación de la estrategia de calidad; esfuerzos que muchas veces se ha expresado en la creación de unidades organizativas dedicadas a la calidad; las propuestas de calidad se han constituido en la base de estudios administrativos con pretensiones de explicación del fenómeno administrativo, llegándose a niveles -en algunos centros de investiga- 
ción- de querer asumir esta vía como la única estrategia de investigación administrativa, y finalmente sin ánimo de agotar las diversas formas de expresión de la importancia que ha tomado la calidad, la enseñanza de la administración ha tomado un sesgo de calidad y productividad en tanto encontramos esfuerzos por hacer de la calidad en el campo de la educación administrativa, la única alternativa de gestión.

En la década de los ochenta y parte de los noventa, la calidad, la calidad total, la calidad y productividad y términos similares han ocupado un espacio relevante en la disciplina administrativa en general y particularmente en otras disciplinas en las cuales a la administración se le asigna importancia significativa, tal es el caso de la educación y la salud entre otras.

La producción intelectual sobre calidad-soporte de los procesos de divulgación e implantación- la constituyen en su mayor parte, propuestas técnica, destacando sus bondades. Escasamente encontramos propósitos explicativos y análisis crítico, cuestión necesaria para avanzar en la construcción de la ciencias administrativa, especialmente si se tiene en cuenta que son propuestas que han avanzado en la práctica, de hecho las tesis sistematizadas por Deming, considerado el padre de la calidad, se sustentan en prácticas en empresas japonesas.

En este trabajo nos proponemos hacer una reflexión crítica sobre el modelo calidad y productividad. Partiendo del supuesto de que éste ha sido un requerimiento de la nueva organización de la producción, motorizada por la necesidad de competencia basada en la producción individualizada sobre la base de las de- mandas particulares del cliente, la cual sustituye la producción en masa. En este contexto la gestión de calidad y productividad no puede ser un modelo en el cual todos ganan, los grandes afectados son los trabajadores y usuarios exciuidos.

\section{Emergencia de la relación calidad-productividad}

La calidad por un lado y la productividad por otro, son conceptos de uso cotidiano en la práctica administrativa, al menos desde que surge la disciplina administrativa, lo novedoso es la incorporación de la relación entre ambos conceptos, es decir, no se trata de cualquier calidad, sino de aquella que tenga vinculación con la productividad y si por tal entendemos (sobre esto parece que hay consenso) el resultado de la relación entre lo producido y los factores usados en la producción, se trata entonces de calidad que permita producir más a menor costo. Tampoco se trata de cualquier productividad, sino de aquella que garantice calidad, que a su vez (siguiendo la reacción en cadena de Deming, al menos hasta aqui) garantice competitividad.

La Calidad y Productividad emerge como un tratamiento sociotécnico en tanto infiere sobre calidad de producción y calidad de vida, por las frases, mejoramiento de los procesos, satisfacción de las necesidades de los clientes, satisfacción del operario, y círculos de calidad (Harrinton, 1988). Entender to que es el modelo de calidad y productividad obliga a estudiar sus orígenes que hacen referencia a Japón y a los Estados Unidos en el final de la Segunda Guerra Mundial. 
Al concluir la guerra como consecuencia del deterioro de los mayores productores de Europa y Asia, Estados Unidos debía cubrir grandes cantidades de producción, razón por lo que se descuidan los atributos que debían poseer sus productos; esto no preocupó a los empresarios norteamericanos porque estaban incorporando nuevas tecnologías, maquinarias, conocimientos, lo que suponia que las metas o propósitos finales estaban relacionadas sólo con la maximización de la producción. En este afán, los directores de planta comentaban que "...de acuerdo a sus experiencias, si se hace avanzar a la producción, se resiente la calidad...", así se pone de manifiesto que se estaba manejando un inadecuado concepto de calidad, y de cómo conseguirla (Deming, 1991:1)

En la industria norteamericana entonces comienza a sentirse la necesidad de mejorar los conceptos e interrogantes en torno al manejo de la calidad. Se introducen asi los elementos de control estadístico de calidad (SQC) para avanzar en el desarrollo del concepto de control de calidad. Asi el gerente de control de calidad de la General Electric de New York, Armand Feigeubaum, acuña por primera vez el término Control Total de Calidad para referirse a un sistema de compatibilidad entre economía de producción y satisfacción de atributos requeridos por los clientes (Mariño, 1991:91).

Se crea sí en las empresas de Estados Unidos un lugar para especialistas en control estadistico de calidad, quienes manejaban la mejora de la calidad "...sólo hasta el nivel en el que las cifras visibles despejen dudas sobre el beneficio econó- mico de seguir mejorando" (Yoshikasu Tsuda, citado por Deming, 1991).

Por otra parte Japón durante el trabajo de su reconstrucción, envía a sus ejecutivos a diferentes partes de Europa y Estados Unidos para conocer procesos de producción de empresas exitosas; de cuya información se ajustarían o adaptarían aquellas prácticas que tuviesen que ver con la reducción de costos, expansión de beneficios y ampliación del mercado. Los resultados de esta búsqueda de información develan que las compañías de mayor éxito son las que determinaban para esa época estándares altos de calidad de sus productos y servicios (Harrinton, 1988).

La relación calidad y productividad es parte de transformaciones que tuvieron lugar en la organización del trabajo en Japón, iniciadas en la Toyota inmediatamente después de la segunda guerra mundial, que según Coriat constituyen "...un conjunto de innovaciones en la organización cuya importancia es comparable a lo que en su época fueron las innovaciones en la organización aportadas por el taylorismo y el fordismo..." (Coriat, 1995:14) y que conducen a la conformación de un modelo, que sólo puede ser comprendido, según Ohno: pensando "al revés" la herencia de occidente (citado por Coriat, 1995:15).

La calidad se convierte para empresas japonesas en un elemento de trabajo diario; y en la búsqueda de referencias acerca del aumento de la Calidad, se incorporaron planes de educación, de instrucción, de capacitación, de incorporación del conocimiento, lo cual sentaba las bases de una sociedad competitiva, 
que sirvió para establecer ciertas prácticas y métodos de desempeño, que unificados en las empresas japonesas dieron como resultado el aumento de la demanda de sus productos, $y$ una filosofia diferente a la occidental en cuanto al manejo de la Calidad.

Señala Drucker (1995:52) que Japón toma por recomendaciones de expertos el invento de la Segunda Guerra Mundial: la capacitación, la cual "...durante los años de la guerra les permitió a los Estados Unidos convertir a su población preindustrial no calificada en trabajadores eficientes, de alta productividad..."señalando que asi mismo los japoneses "...transformaron rápidamente a su gente no calificada y de jornales bajos, en trabajadores altamente productivos pero que seguían ganando bajos jornales y cuya producción podía competir en los mercados desarrollados".

El repunte de la economía japonesa generó grandes expectativas en Estados Unidos y Europa, por la competitividad de sus productos y por ende de la cobertura de los mercados; todo ello basado en el concepto de Calidad de sus productos, que se canaliza con aspectos o enunciados mitificados tales como necesidades y/o satisfacción de las necesidades de los clientes. La consolidación de contenidos en torno a calidad y productividad, la promueven los japoneses cuando involucran aspectos como la participación, cooperación, formación, información sobre procesos, recomendados por Deming en 1950 (Deming, 1989).

Habiendo recuperado relativamente los daños ocasionados por la guerra, la economia japonesa en 1950 aún no podía compararse con la de otros países de- sarrollados. Sus productos no poseían la condición necesaria para dejar de ver el "made in Japan" como sinónimo de imitación barata. Sin embargo al final de la década de los '70, Japón compite en cuanto a su producto internacional bruto con Francia, Inglaterra y Estados Unidos (Vogel, 1981).

Comienza en la década de los 70 en Japón un movimiento encaminado a difundir los conceptos que sobre Calidad se venia experimentando en paises desarrollados. Los directivos de industria integran al consumidor como pilar para lograr objetivos de calidad, así se institucionaliza la frase "necesidades del cliente", que no es más que la atención a ciertas características del producto sugeridas por el cliente. Japón renace con la ruptura de la tradición de ver la calidad como resultado del proceso de producción para tomarlo como Calidad atribuida por el consumidor.

Esto se tradujo en definiciones o en especificaciones técnicas que pasan a ser normas de la empresa. Toda la normativa que cada institución estableció estaba marcada de una revolución cultural con gran carga ideológica. Esta es una de las grandes razones para entender que el éxito de la economia japonesa no se podría trasladas en meras especificaciones técnicas para lograr resultados semejantes en otro tipo de economia. Así lo señala Vogel (1981) y lo confirma Morishima(1989) afirmando que de hecho la economía japonesa, la China o la soviética y el sistema occidental tienen un carácter muy diferente, sería un error tomar una de estas economías como posibles modelos para paises atrasados. 
Japón establece las bases para la apertura de un nuevo modelo de desarrollo a partir de su propia historia. Japón contrapone al método estadounidense de producción de automóviles en cantidades crecientes y variedad de modelos restringidos, proyectando un método de producción basado en fabricar a buen precio pequeños volúmenes de muchos modelos diferentes. Sin embargo, esto abrió la interrogante en el mundo occidental respecto a: ¿cómo elevan los japoneses la productividad cuando las cantidades no aumentan?

El asunto parece sencillo de ver, entendiendo que algunos conceptos entre ellos el de "economía", se interpretan como reducción de costos. Sin embargo esto genera un tipo de confusión, ya que la reducción de personal es un medio para reducir costos, pero esto es un riesgo, ya que el recurso humano es condición para la supervivencia y el crecimiento de un negocio. La explicación a esta controversia se da en la nueva interpretación que le dan los japoneses al concepto de Productividad, viéndola como el repensar de la organización del trabajo en todos sus detalles (Coriat, 1995). De forma tal que la Productividad se asocia en el Japón con producir lo justo, producir en el tiempo necesario, con los recursos necesarios y en las cantidades necesarias, lo que algunos autores entre ellos Ohno(creador del modelo "pequeños volúmenes de muchos modelos") citó como "Justo a Tiempo".

Estos cambios se producen después de la segunda guerra mundial, dando lugar a modelos administrativos denominados gestión de calidad que según sus defensores constituyen un "nuevo cri- terio de gestión" (Deming, 1989:22). Tales modelos aunque con variantes numerosas, dependiendo del autor, tienen como rasgo fundamental el uso de la relam ción calidad-productividad como criterio básico de dirección.

A Deming se atribuye el mérito de la divulgación y generalización de la propuesta de Calidad y Productividad con las fórmulas de reducción de gastos y aumento de los indices de conversión Producción/Insumo; pero existe consenso respecto a que el punto de partida de la consideración de calidad vinculada a productividad se encuentra en Japón, aunque no hay coincidencia respecto a los sujetos que introducen tal relación en la producción, repetimos, erróneamente se le atribuye a Deming las ideas originales sobre la consideración de la calidad vinculada a la productividad, este autor a nuestro juicio, tiene el mérito de la difusión y de la elaboración de una propuesta particular, alimentada de su observación en el trabajo con gerentes y empresarios de la Bell Telephone Laboratories, así como en las experiencias de transformación que se estaban produciendo en Japón después de la segunda guerra mundial.

\section{Calidad y productividad más allá de los objetivos declarados}

La forma en la que Edward Deming concreta la reacción en cadena recoge ciertos sistemas de valores, entre los cuales figura: la constancia, la nueva filosofía, el liderazgo, la lealtad-confianza, la automejora, el equipo, entre otros. Estos valores se conectan a una red de influencias entre sí con propósitos de racionali- 
dad económica del proceso productivo. Se busca someter las acciones y libertad del trabajo a ciertas especificaciones del sistema productivo, con ilusiones de universalidad y de trascendencia del orden social; sin embargo en esencia, mas no en la apariencia, se mantiene el individualismo, la particularidad de intereses y la conservación del orden social vigente de exclusión y elitismo (Reis Silva, 1996).

El discurso de Edward Deming partió de considerar que la empresa mejora el nivel de vida del hombre, y que en los esfuerzos del trabajador por lograr esto, no perdería su trabajo por contribuir a la Calidad y Productividad. Más allá de este propósito está el principio o uno de los 14 puntos de Deming que es la sustitución de la inspección y por ende de la función y responsables de este rol, basándose en que "...cada inspector confía en el otro para que le haga su trabajo". "La responsabilidad dividida quiere decir que nadie es responsable..." (Deming, 1989:24). Entonces nos preguntamos icómo puede haber en una organización un propósito de cooperación, si la nueva filosofía lo excluye o lo confunde en su identidad?, el trabajador se preguntará ¿qué puedo importar yo en este proceso?

La propuesta de calidad y productividad entra en una contradicción en muchos de sus propósitos, tal vez esto apunte a ver qué hay más allá de sus objetivos declarados. En la propuesta de Deming (1989), y revisada por Ishikawa (1990), observamos que existe una contradicción con el fenómeno administrativo básico que suponía Vroon y Deci (1989) relacionado con la psicología del trabajo, según el cual los trabajadores se esfuerzan más cuando pueden tomar decisiones sobre la forma en que desempeñan su labor. En la propuesta de calidad y productividad, esto es lo que no se quiere: que se tomen decisiones sobre cómo realizar los procesos, siendo que éstos están siendo diseñados bajo la forma de mejora continua, acatamiento de normas y especificaciones que viene de los expertos.

Señalamos de la misma forma que la propuesta de la Calidad, no declara, o mantiene como teoría en uso, aspectos relacionados a la supeditación de los intereses individuales, cuando por ejemplo entra en contradicción con lo planteado por Bain (1992) acerca de la solución de los problemas de la empresa y la referencia al desarrollo del sentimiento de que todos los trabajadores son importantes como individuo. En esencia, la propuesta de calidad y productividad sugiere permanentemente la atención a la efectividad de los procesos; y el recurso humano sería así solo una razón para enunciar frases impactantes.

Dentro de las interpretaciones criticas a los propósitos de la propuesta de calidad y productividad encontramos que más allá del propósito de la adopción de posiciones filosóficas de la concepción del mundo, está el enturbiamiento de la visión intra e interpersonal; en el sentido de que la Calidad devela claramente aspectos técnicos, o sea que las acciones de los gerentes de calidad están marcadamente referidas a los procesos, procedimientos, productos y no propiamente en necesidades de crecimiento personal e intergrupal de la masa de trabajadores. La contradicción en este caso implica que se declaran aspectos filosóficos de la calidad y produc- 
tividad los cuales rezan que todos los empleados sin excepción tiene el suficiente potencial para aprender continuamente y mejorar la calidad humana y técnica (Inohara, 1990), sin embargo la preponderancia de las acciones de calidad tienen una orientación eminentemente hacia la mejora de procesos productivos, mejora del trabajo y los procedimientos más que hacia el desarrollo de la visión intrapersonal de los trabajadores.

Por otra parte las sustentaciones técnicas de calidad y productividad hacen referencia al incremento del valor a través de la productividad del trabajo, rememoramos a Carlos Marx en su definición de plusvalía relativa, haciendo énfasis en la disminución del tiempo de trabajo y la correspondiente elevación de la fuerza productiva del trabajador, que implica aumento de su capacidad de producción. Asociada a esta relación la propuesta de calidad y productividad tiene un profundo contenido "praxeológico" como calificó Ludovic (1977) a la teoría científica de Taylor. Se observa que la propuesta de calidad y productividad, como el taylorismo, constituye un conjunto de gulas para la acción práctica de la organización del trabajo en empresas capitalistas.

Contraria a sus puntos básicos o principios fundamentales de liderazgo, lealtad, confianza y continuidad, calidad y productividad se presenta en su transfondo como una propuesta de corte aplicativo pese a las impactantes frases como filosofía, misión, visión, círculos de calidad, etc. La naturaleza aplicativa es notoria analizando muchos de sus contenidos; el más importante es el que se asocia como el propósito concreto de "aumento de la productividad", de acuerdo al quinto principio para la salida de la crisis planteado por Deming. Cuando se hace referencia a la naturaleza aplicativa de esta propuesta también se argumenta su pensamiento "utilitarista" que la nutre. El utilitarismo está expresado en el mismo concepto de "hedonismo" que caracterizó el sistema de valores en el que sustentara el paradigma clásico. Es decir, la calidad y productividad lleva implícito la exhortación de que todo el mundo debería preguntarse a sí mismo "¿qué es lo que se ha hecho en el día para avanzar en su conocimientos sobre el trabajo y mejorar la habilidad... para estar más satisfecho en la vida?" (Deming, 1989:39). Esta recomendación está absolutamente en correspondencia con al explicación del desarrollo social mediante los motivos de la conducta, aquéllos que llevan al hombre a la "búsqueda del placer y supresión del dolor" como expresión conceptual de la doctrina hedonista (Ludovic, 1977). Más aún, esto da cuenta de una forma más de confusión entre dominación y autodominación con independencia; la autodominación es un efecto directo de aquella exaltación del sentido hedonista que no alcanza a mostrar la verdadera conjunción de las técnicas de control de procesos naturales y las técnicas referidas al control del comportamiento de los hombres (Dávila, 1997).

Más allá de los propósitos declarados de la propuesta de calidad y productividad encontramos la influencia de la doctrina del liberalismo económico cono se observa que "el dinero es la principal razón económica" como consecuencia de la reproducción del capital, que se concreta en la Globalización 
del mercado mundial (fin de la propuesta de calidad y productividad). Las compañías transnacionales en desmedro de los gobiernos y/o Estados nacionales, se posesionan del poderío económico financiero, apoyado por organismos internacionales como el Banco Mundial, el Banco Interamericano de Desarrollo, el Fondo Monetario Internacional; así la Privatización como desestatización, son componentes estructurales de la propuesta de calidad y productividad que cada vez más, da cuenta de una legítima concepción neoliberal.

Basta un poco de buen sentido para notar en esta propuesta la permanencia de la ideología de dominación, cuando en unas exhortaciones tan enmascaradas como filosofía de gestión, actitudes positivas, valores, participación, información y satisfacción, se cubre el verdadero carácter de lo que es "prosperidad del patrón e interés del capital"; para lo cual el verdadero papel del trabajador es dejarse conducir u organizar con el fin de ser más productivo en el trabajo.

Lo que en realidad plantea la calidad y productividad no es la conducción de los procesos bajo la filosofía de "cero defectos", sino una alternativa muy bien establecida para resolver el control del trabajo alineado, que permita la "supremacia del poderío del capital", mucho más aplicable ahora cuando el trabajo muestra ser verdaderamente parte del capital en tanto que la nueva legislación del trabajo indica una predominante parte del capital comprometida con la permanencia y retiro de un trabajador en la empresa.

\section{Calidad y productividad: nuevo modelo de organización del trabajo}

Ciertamente la calidad y productividad se podría interpretar con el análisis del trabajo y/o los procesos del trabajo u organización del trabajo; es una propuesta válida para concebir un trabajo, un trabajador y una empresa productiva. Sin embargo, el análisis del trabajo bajo cualquier propuesta no es tarea fácil, ya que las implicaciones de una nueva concepción de trabajo tienen que ver con estructura de cargos y funciones, funciones de línea y staff, relaciones intra e interdepartamentales, y decisiones estructurales, y con la concepción del trabajador formado, entre muchos otros aspectos.

La propuesta de calidad y productividad representa el cambio de la concepción del trabajo basado en la especialización del operario para referirse a un trabajador multifuncional (Pérez, 1990) que conozcan el proceso desde su inicio hasta la culminación; capaz de hacer modificaciones necesarias como parte de la exigencia del mejoramiento continuo. Sin embargo no todas las aplicaciones de la propuesta han significado una concepción similar en cuanto a organización del trabajo se refiere. Por ejemplo la calidad y productividad que se implementa en Estados Unidos estuvo representada por un método de trabajo en el que no se consideró cantidad ni variedad del mismo, sino específicamente evitar errores o desperfectos como vía de reducir costos. Los estadounidenses trabajan para la gestión de calidad con una organización del tra- 
bajo diferente a los japoneses, basada en actividades desarrolladas en diferentes funciones que van desde la investigación de las necesidades hasta el servicio de postventa. De esta forma la calidad y productividad en Norteamérica ligeramente ha sido ubicada en departamentos línea o áreas técnicas, de producción con un enfoque tecnológico más que integracionista; los norteamericanos han prescindido de la conformación de estructuras adecuadas y ha sucedido que las diferentes funciones buscan sus propios resultados individuales en detrimento de los resultados de la empresa.

Esto ha generado otros inconvenientes y es llegar a determinar cuál es la unidad o departamento apropiado pata orquestar la calidad y productividad. A lo largo de casi medio siglo de estar hablando de calidad y productividad, los Estados Unidos no han recorrido las mejores experiencias en relación a la implementación de esta propuesta.

Ya lo había advertido Feigenbaun de la General Electric (citado por Mariño, 1991) que la mejor organización del trabajo para este acometido estaba en aquella que garantizara la coordinación de todos los esfuerzos en todas las divisiones de la empresa; pero recomendó respaldar la calidad por una función gerencial organizada para tal fin que le imprimió el carácter de función especializada que requería profesionales especializados. Así el enfoque occidental hacia la implementación de calidad y productividad implicó la creación de grandes estructuras organizacionales como gerencias, divisiones, departamentos de control de calidad; esto generó una incertidumbre de quien debia asumir el mayor compromiso y termina en falta de involucramiento y de participación de todos los niveles jerárquicos.

Además de esta limitación, el mundo occidental se consigue con un problema y es que por la propia ausencia de participación e involucramiento no se reconocía a quien le competía la educación, formación y capacitación en el tema, lo que deriva en una adjudicación del conocimiento de calidad solo a los privilegiados especialistas (Mariño, 1991).

Pensando al revés, los japoneses desarrollaron un concepto de calidad y productividad que involucraba toda la empresa, es decir con una concepción diferente en cuanto a método de producción, el cual estaba basado en la producción a buen precio, de pequeños volúmenes de muchos modelos diferentes. Lo que requería un trabajador multifuncional, no especializado, que igual que en el esquema clásico implica racionalización del trabajo, así sea una concepción diferente del trabajador, pero que ambos tienen su transfondo operacional, es que al obrero no tenga poder sobre la producción, no tenga poder de negociación.

Esto da la idea de una nueva concepción del trabajador y del trabajo que cumple la misma aspiración en cuanto al control de la cantidad de información que deba manejar el trabajador sobre el sistema porductivo total. $Y$ es solo la posibilidad del conocimiento de fallas y aciertos en los procesos, dándole al trabajador solo conocimiento útil a la implementación de la técnica.

Sin embargo la implementación de calidad y productividad en el Japón imprime una concepción de trabajo y trabajador con más conocimiento sobre el tema, 
porque la empresa japonesa practica el enfoque de involucrar a cada colaborador de cada división, en la construcción de la calidad, asi el sentido de participación es valorable, porque no es exclusividad de los especialistas. Además esto ha hecho posible favorablemente que las personas que realicen el trabajo bajo estas especificaciones se autocontrolen y construyan la calidad, para lo cual han sido formadas no necesitan el cargo del supervisor para determinar si pasa o no el producto a la siguiente fase.

Las organizaciones en estos casos van constituyéndose en organizaciones más planas que verticales, donde los cargos de supervisión van desapareciendo, y por ende se da la cercanía de los problemas a sus soluciones, porque mientras menos niveles jerárquicos, más directos son los diagnósticos y más rápidas las respuestas. Sin embargo, la critica de cuenta de que este modo de trabajo es disciplinario, y en sus intensiones, igual que el trabajo de la gran empresa de comienzos de siglo; ahora la vigilancia (con tanta carga negativa para la concepción humanista del trabajo) pasa a ser un operador económico decisivo, en la medida en que es a la vez una pieza interna en el aparato de producción y un engranaje especificado del poder disciplinario (Foulcault, citado por Dávila, 1994).

La crítica considera que la calidad y productividad desde el punto de vista de la concepción de la organización del trabajo, ha fundamentado una nueva forma de legitimación de la dominación cuando se le da a la productividad un sentido de recompensa, o sea que la idea de rendimiento máximo en el trabajo tiene un matiz benévolo y justifica el control y la vigi- lancia del comportamiento del trabajador. Esto hace, según Dávila (1994) que se conciba a la calidad y productividad como el más refinado modo de explotación del hombre por el hombre.

De alli que las apreciaciones críticas de la propuesta de calidad y productividad generalizan que ésta es la traducción o versión actual con algunas modificaciones en su contenido, de la propuesta que afloró a comienzos de siglo como técnicas y herramientas fácilmente aplicables, que se constituyó, igual que ahora, en una filosofía del obrero y del trabajo, cuando en esencia era una forma de dominación más. Por ello, la crítica al discurso taylorista de comienzo de siglo, es válida para referirse a la calidad y productividad, señalando que ambas tienen un marcado origen ideológico que más que reflejar una forma explicativa de una realidad (teoria) o un modelo dentro del cual se le da respuestas a una serie de problemas de la Administración (paradigma) parte de una realidad empírica, donde se asume como verdadero to observable. Apenas si se infiere que el problemas de dirección se asume desde distintas realidades históricas, desfavoreciendo el valor real de al aplicabilidad y extensión de la propuesta, porque ésta se hace distinta dependiendo del escenario, y siempre va a tener un elemento determinante que son las condiciones materiales de existencia, propias del sistema capitalista.

\section{Conclusiones}

La Calidad Total en este estudio se descubre como la racionalidad del proceso productivo, que refuerza mecanismos para disminuir el poder del empleado $u$ 
obrero calificado sobre la producción, incrementando la intensidad del trabajo, cambiando la especialización por desespecialización, así como el modelo de producción, alrededor de lo cual están los propósitos de incrementar la ganancia y la expansión del capital. Igual que los enfoques tradicionales del pensamiento administrativo (mecanicista, burocrático y estructural), la calidad y productividad se caracteriza por conceptos que están relacionados con la disminución de costos e incremento de beneficios, que implican la ejecución de procesos con menor consumo de materias primas e insumos, para llegar al fin último de posesión de mercados internacionales.

Hasta ahora muchas de las definiciones de la calidad y productividad que han hecho investigadores, instituciones, intelectuales, academias y empresarios, la presentan como una teoría, sin corresponderla con el tiempo y lugar en el que se adopte. Lo que implica que pueda calificarse como definiciones abstractas con intención de universalidad en un todo de acuerdo a la posición crítica de Joaö Reis Silva (1996).

Epistemológicamente se interpreta que pese a que la calidad y la productividad se sustenta en un vocabulario observacional entendible y manejable (productos, procesos, mercados globalizados, nuevas tecnologías, clientes, etc.) las reglas de correspondencia con sus criterios y constructos teóricos revelan una aproximación a la orientación hacia la acumulación que busca someter todos los ámbitos de la vida organizacional y de un país, a un sistema productivo que permita preservar un determinado orden social; en contradicción con la naturaleza de los fe- nómenos sociales y educativos con niveles de transformación, de inclusión en vez de exclusión, de participación y/o sentido de pertenencia y no de elitismo que es lo que domina el orden social vigente.

Existe una interpretación de la ideología liberal en los constructos teóricos de los que emerge la calidad y productividad, cuando ésta hace énfasis en la forma, los métodos, medios materiales y financieros, en los que se supone que la crisis de la calidad de producción, de la vida $y$ de trabajo en un país $u$ organización, nadie antes la hubiese concebido como propósito, y utiliza por demás un contenido filosófico, para llegar al condicionante del nuevo orden mundial: la Globalización. La calidad y productividad es un condicionante además, de las nuevas tareas de la tecnología.

Hemos encontrado que en el análisis de su basamento filosófico emerge una forma que busca la consolidación del sistema capitalista a nivel mundial. Se logra en el presente estudio, mostrar el papel ideológico, condicionante de este sistema; no obstante no contiene la calidad y productividad la posibilidad teórica que sea capaz de explicar los fenómenos administrativos o la realidad administrativa desde el punto de vista de la correspondencia de contenidos, con teorías y con suposiciones teóricas amplias, que permitan formular planeamientos teóricos analíticos de largo alcance y validez general. Es decir que más que una teoría o una propuesta que genere conocimiento administrativo y que alcance a marcar la evolución del pensamiento administrativo, la calidad y productividad es apenas el conocimiento del funcionamiento final de las cosas y procesos, propuesto por 
algunos autores como reglas técnica donde solo se define el "cómo" y no el "por qué" de los procesos.

La calidad y productividad entendida solo como propuesta no está orientada hacia la obtención de verdaderos conocimientos de la realidad administrativa, porque alcanza apenas el manejo de contenidos teóricos útiles para elevar la productividad, sin medir efectos de largo alcance; además sus premisas (que por analogía sería su escaso sistema de valores) solo son ideología, que hacen que el rigor científico quede subordinado a una contagiante euforia por frases, exhortaciones y celebridades, pero no dan cuenta o no explican los problemas sociales, administrativos $u$ organizacionales.

Todas las personalidades del mundo académico o empresarial que se han dado a la tarea de ahondar y resaltar el contenido de la propuesta de calidad y productividad, sus aplicaciones y experiencias en paises desarrollados, se encierran en un componente técnico para la acción del capitalismo en la mira de perpetuar el control total sobre el trabajo; asumiendo el control sobre los procesos se infiere que se pueden resolver los problemas de una clase elitesca capitalista, y por demás se consigue perpetuar las diferencias de clases, en un marco de condiciones sociales en el que se es indiferente al concepto de lucha, toda vez que la clase trabajadora cree en los conceptos filosóficos que han hecho furor en la propuesta de calidad y productividad, como satisfacción, calidad de vida, formación, participación, etc.
En este estudio se marca, además de ese carácter alienante, el carácter utilitarista de la propuesta. Se han traducido las posiciones críticas del taylorismo a esta propuesta; $y$ se puede señalar que la calidad y productividad se fundamenta igualmente en un pragmatismo vulgar que reza que lo que es útil al capitalismo, es útil a los demás.

La propuesta de calidad y productividad, finalmente permite reconocer la función ideológica propia de la filosofía del capitalismo, en sus propósitos de mostrar fines económicos como el mejoramiento de la sociedad por medio del mejoramiento continuo, la perpetuidad y naturalidad de la situación social del sistema capitalista. Así, esta propuesta pudiera semejarse a una instrumentaria teórico-práctica que la empresa capitalista utiliza para sistematizar el rol de la Administración de empresas con su interior, sustentando las relaciones que mantiene con el sistema en el que se sostiene.

\section{Bibliografía citada}

Bain, David (1992). Productividad, McGraw Hill. México.

Coriat, Benjamin (1995). Pensar al Revés. Trabajo y Organización Capitalista. Editorial Siglo XXI. España.

Dávila, Jorge (1994) . Critica Parcial de la Calidad Total. Dpto. de Sistemología Interpretativa. U.L.A. Mérida.

Deming, Edward (1989). Calidad, Productividad y Competitividad: la salida de la crisis. Diaz de Santos. Madrid.

Drucker, Peter (1995). Gerencia para el Futuro. Editorial Norma. Colombia. 
Harrinton, James (1988). Cómo incrementar la Calidad y Productividad en su empresa. Mc Graw Hill. México.

Inohara, Hideo (1990). Desarrollo de Recursos Humanos en la Compañias "Japonesas". Fondo de Investigación para el Mejoramiento de la Productividad. (Documento).

Ishikawa, Kaoru (1990). ¿Qué es la Calidad Total? Editorial Norma. Colombia.

Jurán y Grina (1995). Análisis y Planeación de la Calidad en Bienes y Servicios. Editorial Limusa. México.

Ludovic, Valmis (1977). El Productivismo en las Teorias Administrativas. Contexto Editores. Caracas.

Mariño Hernando (1991). Gerencia de la Calidad Total. Tercer Mundo Editores. Colombia.
Morishima, Michio (1989). ¿Por qué ha triunfado Japón? Editorial Grijalbo. Cara cas.

Pérez U., Rafael (1990). La Calidad, eje de la Empresa Japonesa. Editorial Siglo XXI. España.

Reis Silva, Joäo (1996). Calidad Total en la Educación. Revista del Centro Experimental de Estudios Latinoamericanos (CEELA). Vice-Rectorado Académico. Universidad del Zulia. Maracaibo. Venezuela.

Vogel, Ezra (1981). Japón No.1. Editores Técnicos Asoclados. España.

Vroon y Daci (1989). Psicología del trabajo. Editorial Mc Graw Hill. México. 